\title{
Substructure Depletion in the Milky Way Halo by the Disk
}

\section{Citation}

D'Onghia, Elena, Volker Springel, Lars Hernquist, and Dusan Keres. 2010. "SUBSTRUCTURE DEPLETION IN THE MILKY WAY HALO BY THE DISK." The Astrophysical Journal 709 (2): 1138-47. https://doi.org/10.1088/0004-637x/709/2/1138.

\section{Permanent link}

http://nrs.harvard.edu/urn-3:HUL.InstRepos:41381808

\section{Terms of Use}

This article was downloaded from Harvard University's DASH repository, and is made available under the terms and conditions applicable to Open Access Policy Articles, as set forth at http:// nrs.harvard.edu/urn-3:HUL.InstRepos:dash.current.terms-of-use\#OAP

\section{Share Your Story}

The Harvard community has made this article openly available.

Please share how this access benefits you. Submit a story.

Accessibility 


\title{
SUBSTRUCTURE DEPLETION IN THE MILKY WAY HALO BY THE DISK
}

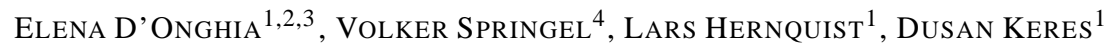 \\ 1-Harvard-Smithsonian Center for Astrophysics, 60 Garden Street, Cambridge, MA 02138 USA \\ 4-Max-Planck-Institute for Astrophysics, Karl-Schwarzschild-Str. 1, 85740 Garching, Germany
}

\begin{abstract}
We employ numerical simulations and simple analytical estimates to argue that dark matter substructures orbiting in the inner regions of the Galaxy can be efficiently destroyed by disk shocking, a dynamical process known to affect globular star clusters. We carry out a set of fiducial high-resolution collisionless simulations in which we adiabatically grow a disk, allowing us to examine the impact of the disk on the substructure abundance. We also track the orbits of dark matter satellites in the high-resolution Aquarius simulations and analytically estimate the cumulative halo and disk shocking effect. Our calculations indicate that the presence of a disk with only $10 \%$ of the total Milky Way mass can significantly alter the mass function of substructures in the inner parts of halos. This has important implications especially for the relatively small number of satellites seen within $\sim 30 \mathrm{kpc}$ of the Milky Way center, where disk shocking is expected to reduce the substructure abundance by a factor of 2 at $10^{9} \mathrm{M}_{\odot}$ and 3 at $10^{7} \mathrm{M}_{\odot}$. The most massive subhalos with $10^{10} \mathrm{M}_{\odot}$ survive even in the presence of the disk. This suggests that there is no inner missing satellite problem, and calls into question whether these substructures can produce transient features in disks, like multi-armed spiral patterns. Also, the depletion of dark matter substructures through shocking on the baryonic structures of the disk and central bulge may aggravate the problem to fully account for the observed flux anomalies in gravitational lens systems, and significantly reduces the dark matter annihilation signal expected from nearby substructures in the inner halo.

Subject headings: Galaxy: disk - Galaxy: formation - Galaxy - dynamics - Galaxy: structure
\end{abstract}

\section{INTRODUCTION}

In the cold dark matter (CDM) scenario, structure grows hierarchically, with small objects collapsing first and continuously merging to form larger and larger bodies (White \& Rees 1978). Numerical simulations have tracked the evolution of this process, beginning at early times with objects having masses comparable to that of the Earth (Diemand et al. 2005), and progressing through many orders of magnitude in mass to the scales of galaxies, galaxy clusters, and cosmic largescale structure (e.g. Ghigna et al. 2000; Springel et al. 2005). In the Milky Way, the cumulative number $N$ of dark matter satellites of a given mass $M_{\text {sat }}$ is predicted to scale with the subhalo mass as $N \propto M_{\text {sat }}^{-0.9}$ (Springel et al. 2008b). Because the subhalos hosting the satellite galaxies formed early, when the Universe was dense, the smaller structures are thought to be resilient to tidal disruption and the simulations predict that $\sim 100$ with maximum circular velocity larger than $20 \mathrm{~km} \mathrm{~s}^{-1}$ should survive to the present day for a halo like that of the Milky Way (Klypin et al. 1999; Moore et al.|1999; Diemand et al. 2008; Springel et al. 2008b; Kravtsov 2009).

However, these estimates of substructure abundance at different locations within a halo, from the solar neighborhood to the outskirts of the Milky Way, are based on simulations that include only dark matter and do not account for ordinary baryonic material (but see Dolag et al. 2008). This means that any dynamical coupling between dark matter substructures and the luminous components of galaxies has been ignored, even though this may alter the substructure abundance. A precise understanding of the impact of baryons on the substructure abundance is important to correctly assess the relevance of substructures for the evolution of galaxies, and for attempts

\footnotetext{
2 Marie Curie fellow; edonghia@cfa.harvard.edu

${ }^{3}$ Institute for Theoretical Physics, University of Zurich, Winterthurerstrasse 190 CH-8057 Zuerich, Switzerland
}

to detect dark matter through gravitational lensing or annihilation radiation.

For example, recent studies suggest that cosmological infall of dark satellites through the disk of a typical galaxy could induce bars and multi-armed spiral patterns in disks (Dubinski et al. 2008). According to these simulations, the main agent producing transient features would be satellite passages through the inner part of the disk. Indeed, it is speculated that the passage of a satellite through the disk induces a localized disturbance that could grow by the mechanism termed swing amplification (Toomre 1981). Because the tidal effects of the satellites are generally small, this process is distinct from interactions thought to be responsible for granddesign spirals like M51 (Kazantzidis et al. 2009). The mechanism proposed by Dubinski et al. (2008) is closer in spirit to the original ideas suggested by Julian \& Toomre (1966) in which an object within a disk excites a response that is subsequently amplified. Unlike the interactions involving from large visible satellite galaxies, we are more interested in the process produced by the continuing bombardment of a galactic disk by the dark matter halo inhomogeneities that will keep on exciting ragged spiral structures.

Here, we investigate the coupling between dark matter substructures and the luminous components of galaxies. We consider tidal processes that lead to a depletion of the substructures orbiting in the inner regions of a galaxy by the presence of a disk and we discuss its astrophysical implications.

Naively, one might expect that the luminous components of galaxies should play only a minor role in affecting substructures because most of the mass is in the dark matter. However, some gravitational interactions between bodies, especially those involving tidal forces, depend not on the relative masses of objects, but on their relative densities. Whereas the dark matter is thought to be nearly collisionless, baryons can dissipate energy and angular momentum and collapse to high 
densities. This must have been the case for the baryons in the disk of the Milky Way, which is far more concentrated in its inner parts than the dark matter that makes up the surrounding smooth halo. Thus, it is plausible that the baryons could have an impact on the dark matter substructures despite their small contribution to the overall matter density of the Universe, motivating our investigation.

Specifically, we carry out a number of fiducial numerical experiments in which we adiabatically grow a disk in a highresolution collisionless simulation of a Milky-Way sized halo. This enables us to examine the impact of e.g. disk shocking on dark matter substructures that pass through the disk. We also follow the orbits of substructures in the high-resolution dark matter simulations of the Aquarius project, allowing us to identify the parameters and frequency of encounters with an assumed disk in the halo. This provides an independent check of our isolated disk-growth simulations, and also enables an investigation of the consequences of different modeling assumptions.

In $\$ 2$, we discuss the numerical set-up of our disk-growth simulations, and in $₫ 3$ we present the results. In $\$ 4$ we compare with analytic estimates of the disk shocking effect based on tracking satellite orbits in cosmological simulation of halo formation. Finally, we discuss the implications of our results in $\S 5$

\section{NUMERICAL METHODS}

Our simulations were performed with GADGET3, a parallel TreePM code developed to compute the evolution of dissipationless dark matter systems. A detailed description of the code is available in the literature (Springel 2005; Springel et al. 2008a). Here, we note its essential features.

GADGET3 is a cosmological code in which the gravitational field on large scales is calculated with a particle-mesh (PM) algorithm, while the short-range forces are provided by a treebased hierarchical multipole expansion, such that an accurate and fast gravitational solver results. The scheme combines the high spatial resolution and relative insensitivity to clustering of tree algorithms (Bouchet \& Hernquist 1988) with the unmatched speed and accuracy of the PM method to calculate the long range gravitational field. Pairwise particle interactions are softened with a spline of scalelength $h_{s}$, so that they are strictly Newtonian for particles separated by more than $h_{s}$ (Hernquist \& Katz 1989). For our applications, the gravitational softening length is kept fixed in comoving coordinates throughout the evolution of our halos.

\subsection{Setting the initial conditions}

Presently, galaxy formation is not well-understood and so it is not possible to perform an ab initio cosmological simulation that combines the evolution of dark matter and baryons and produces an object that closely resembles the Milky Way. For this reason, we instead employ simulations in which we follow the growth and subsequent gravitational collapse and virialization of dark matter halos self-consistently, but include the gravitational contribution from luminous galaxies in an idealized manner. In particular, we represent the baryons of a galaxy as a fixed disk potential, added to a dark matter simulation with parameters and temporal behavior chosen to match observational and theoretical constraints. This enables us to study the dynamical coupling between luminous galaxies and substructures in their dark matter halos.

In our procedure, we first evolve cosmological simulations with dark matter only and locate halos with properties at the present day similar to those of the Milky Way. The halo described in detail below was originally selected within a low resolution, dark matter only simulation run in a concordance, $\Lambda$-dominated universe with parameters $\Omega_{m}=0.27, \Lambda=0.73$, $h=0.7, \sigma_{8}=0.8$, and $\Omega_{b}=0.044$. These cosmological parameters are consistent with the current set of constraints within their uncertainties, in particular those from the WMAP 1- and 5-year data analysis (Komatsu et al. 2009).

The large dynamic range involved in cosmological simulations aimed at resolving the scales of halos like the one presented here calls for techniques that concentrate the computational power on the object of interest. This is achieved by a zoom-technique, where a large-scale simulation is done at low resolution and the relevant regions are identified within it, e.g. a Milky Way-sized halo such as in this work. The simulation can then be repeated with much higher resolution in this region.

The size of the box we employed, $10 h^{-1} \mathrm{Mpc}$ on a side, is quite small and starts to become non-linear at the present epoch, but it is still sufficient to provide realistic torques and accurate formation histories of Milky-Way sized halos. The virial mass of the halo we selected at $z=0$ to be resimulated at higher resolution is $5.5 \times 10^{11} h^{-1} \mathrm{M}_{\odot}$ (the virial mass is here measured within a radius $R_{\mathrm{vir}} \sim 160 h^{-1} \mathrm{kpc}$, enclosing an overdensity of $\sim 96$ times the critical density (Bryan \& Norman 1998)).

Dark matter particle masses in the high resolution regions are $5.5 \times 10^{5} h^{-1} M_{\odot}$, and the force resolution, i.e. the gravitational softening, is $200 h^{-1}$ pc. In total there are $\sim$ $10^{6}$ particles within the virial radius. With our choices for particle number and softening, the smallest subhalos resolved have typical masses of $\sim 10^{7} M_{\odot}$. The chosen halo has a merger history and spin parameter reasonably representative of the global population of halos in equilibrium (D'Onghia \& Navarro 2007). The halo was selected with the only criterion that the redshift of the last major merger was $z \approx 1.5$ and that there are no halos of similar or larger mass within a few virial radii (a major merger is defined here as having a 4:1 mass ratio). The halo spin parameter is 0.04 at $z=0$, close to the average value $\approx 0.035$ for halos in large cosmological simulations (Bett et al. 2007).

\subsection{Putting a disk inside the galactic halo}

We began the cosmological simulation at high resolution in the region of interest at an early time and evolved it forward to the present day (and beyond), saving the state of the system at many intermediate times. We extracted the galaxy halo at the present time from the cosmological simulation, including all particles within $500 h^{-1} \mathrm{kpc}$ of the galactic center and then repeated the simulations, but adding the potential from a disk to the halo starting at a specific time. We repeated this procedure by varying both the manner in which the galaxy was included as well as its properties.

We have modeled the disk potential according to Kuzmin 1956):

$$
\phi(R, z)=-\frac{G M}{\sqrt{R^{2}+\left(a+\sqrt{z^{2}+b^{2}}\right)^{2}}},
$$

where $M$ is the disk mass, $a$ is the scale-length of the disk and is taken to be $4.55 h^{-1} \mathrm{kpc}$, and $b$ is the disk height, assumed to be $260 \mathrm{pc}$. With these choices, this potential matches that of the Galactic disk (Johnston et al. 1995). We have tested different prescriptions to place the disk inside the halo: we either 


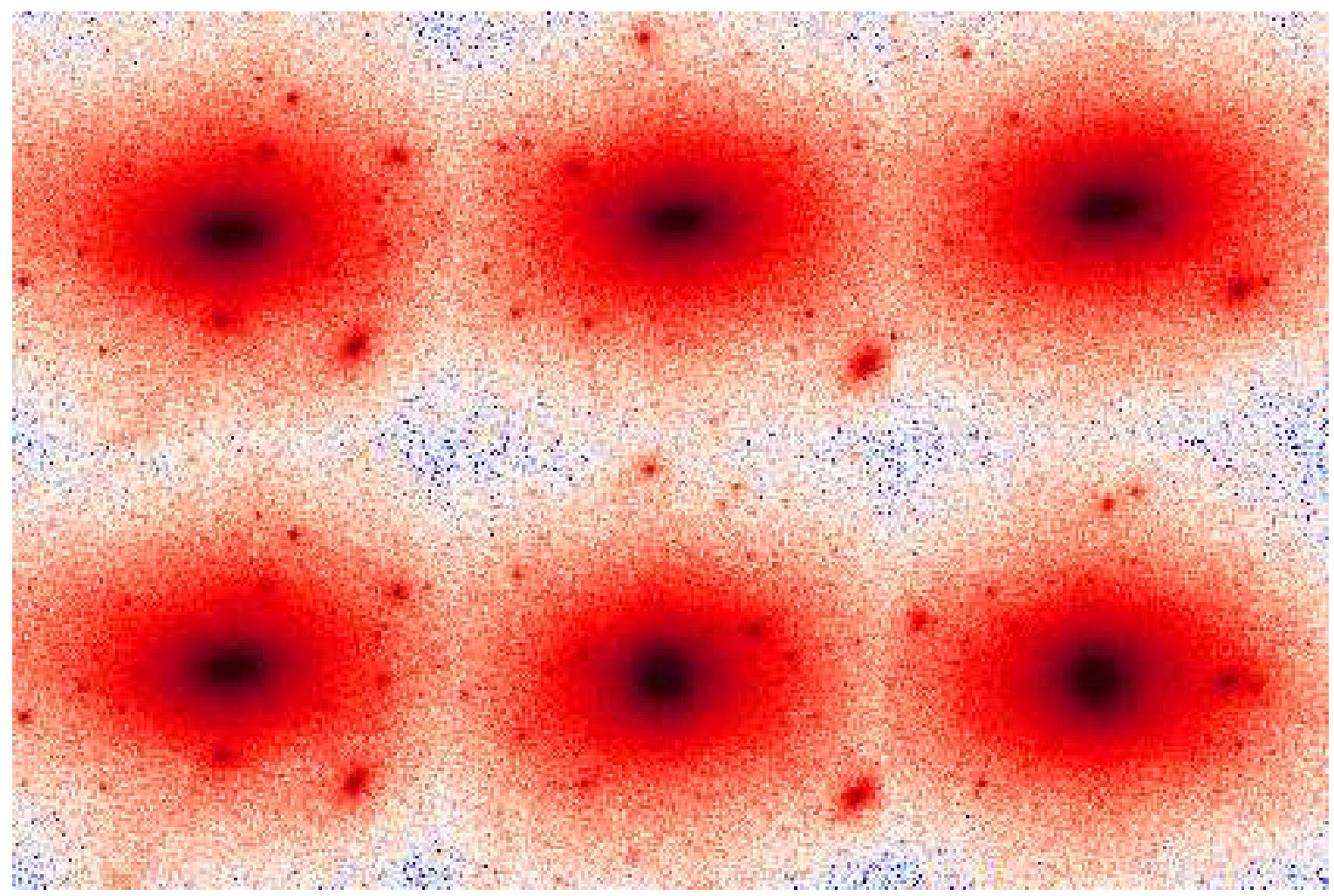

FIG. 1.- Dark matter distribution within $60 \mathrm{kpc}$ of the center of the dark matter halo in two simulations, each at three different times. The columns are the initial state (left column; identical in both cases), after 3.7 Gyrs (middle column), and after 5.5 Gyrs (right column). Top row is a simulation with no disk, bottom row is one where a disk with mass 0.1 times the dark halo was included. For the experiment with a disk, the disk was grown adiabatically over the initial 2 Gyrs to its final mass.

centered on the halo center of mass, or on the particle where the halo potential is a minimum. In the former method we adopted an iterative method to locate the maximum density and centered the disk there. The different procedures gave similar results; for the final runs analyzed in this paper we adopted the minimum potential approach.

In the simulations with disks, we grew the galaxy slowly (adiabatically) for 2 Gyrs to its full mass to avoid initial transients. In our fiducial case, the disk grows to a mass of $1 / 10$ th of that of the halo within its virial radius, but we also performed different simulations altering the properties of the luminous galaxy. In particular, we varied the mass of the disk relative to that of the dark matter halo, its radial extent, and the growth timescale of the disk. In general, we see the heating processes becoming stronger as the mass of the disk or its scale length are increased, consistent with analytic expectations (see below).

We investigate the abundance of dark matter substructures inside the halo as measured by the SUBFIND algorithm (Springel et al.2001). All our substructures consist of particle groups that are gravitationally self-bound and are overdense with respect to the local background. Every simulation particle can be part of only one subhalo. We count substructures down to a minimum of 20 bound particles.

\section{RESULTS FOR DISK-GROWTH SIMULATIONS}

The generic outcome of our experiments is illustrated in Figure 1] which shows the appearance of halos at various times for cases in which we ignored the contribution from a disk (top row) and included it (bottom row). From left to right, we show the dark matter distribution initially and then after 3.7 and 5.5 Gyrs. The simulations shown in this figure assumed a disk with a final mass of $5.5 \times 10^{10} h^{-1} \mathrm{M}_{\odot}$, i.e. $10 \%$

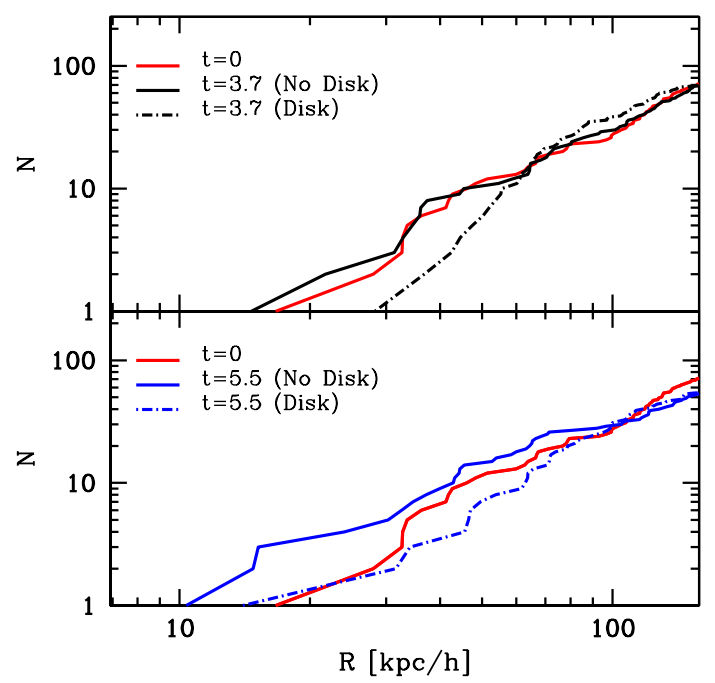

FIG. 2.- Cumulative number of subhalos as a function of distance from the center of the host halo, with and without the disk.

of the dark mass within the virial radius, appropriate for the Milky Way.

As is visually apparent in Figure 1 the dark matter distribution is significantly influenced by a disk. Within 2 Gyrs after reaching its final mass, the disk is able to erase most of the substructures inside the inner few tens of kpc of the halo. Because this timescale is short compared to the age of the Universe and the evolutionary timescales of real galactic disks, we expect that few, if any, low mass substructures would actually be orbiting the innermost regions of a large spiral galaxy.

This is made more explicit in Figure 2, where we compare 


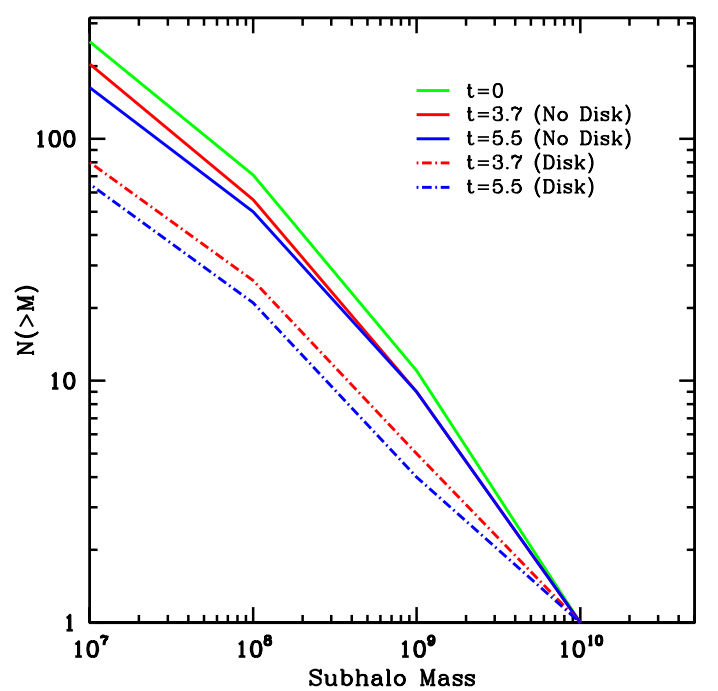

FIG. 3.-Cumulative number of subhalos above a given mass limit, for the evolved simulations with and without a disk within two virial radii. At late times, the disk can reduce the substructure abundance by nearly a factor of two.

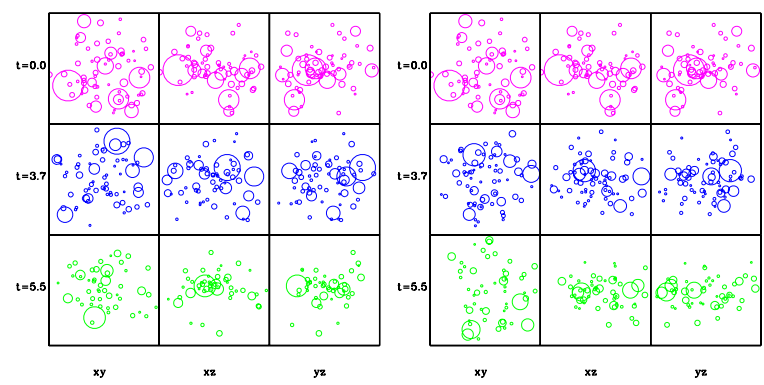

FIG. 4.- Spatial distribution of substructures within $150 h^{-1} \mathrm{kpc}$ of the galaxy center, comparing the case when a disk is not included (top set of panels) with the case where disk is included (bottom set of panels). For both we show the distribution at the initial time $t=0$ (top rows) and after $3.7 \mathrm{Gyrs}$ and 5.5 Gyrs, respectively (middle and bottom rows). Circle areas are proportional to the substructure masses.

the radial cumulative abundance of substructures between the runs with and without disks. As time goes by, the depression of the subhalo function in the disk case increases, in proportion to the larger number of disk passages that have occurred. This substructure depletion is particularly strong in the inner parts of the halo, even though an effect is also noticeable in the outer parts. In Figure 3, we plot instead the cumulative substructure count as a function of mass, comparing again the simulations with and without a disk at two different times after the start of the simulations. There is up to a factor of $\sim 2$ reduction in the substructure abundance, and the effect is approximately independent of substructure mass if we discard the measurement for the most massive bin, which is populated by only one object and therefore allows no statistical conclusions.

The depletion of the substructure abundance is also reflected in the spatial distribution of substructures, which we show in Figure 4 at different times for the cases without a disk (top) and when a disk with a final mass equal to $10 \%$ of the final halo mass included (bottom). The areas of the symbols are proportional to satellite mass.

Figures 2, 3 and 4 confirm that a disk accelerates mass loss by satellites, altering the subhalo mass function. Overall, the

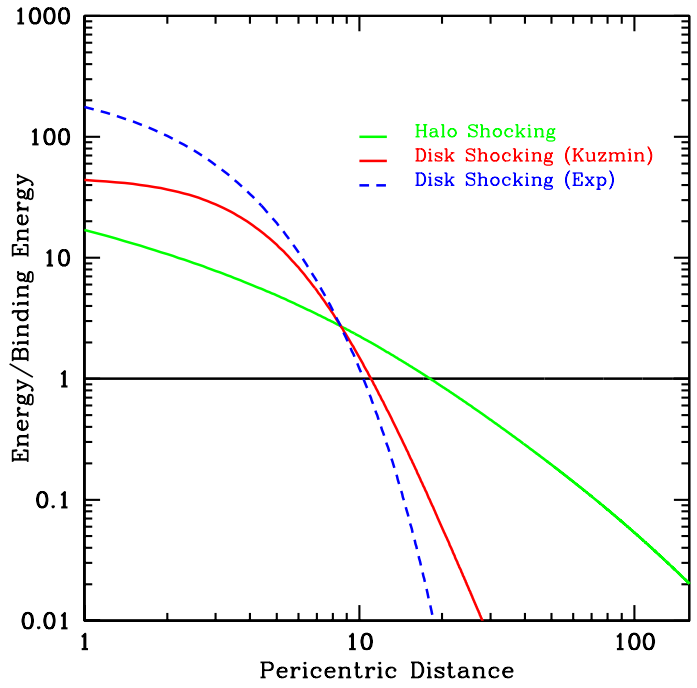

FIG. 5.- Energy of a satellite of $10^{7} \mathrm{M}_{\odot}$ as compared to its binding energy being affected by disk shocking produced by an exponential disk (blue dashed line), by a Kuzmin disk as in our simulations (solid red line), and by tidal shocking from the halo (green solid line) for different pericentric distances of the satellite orbit.

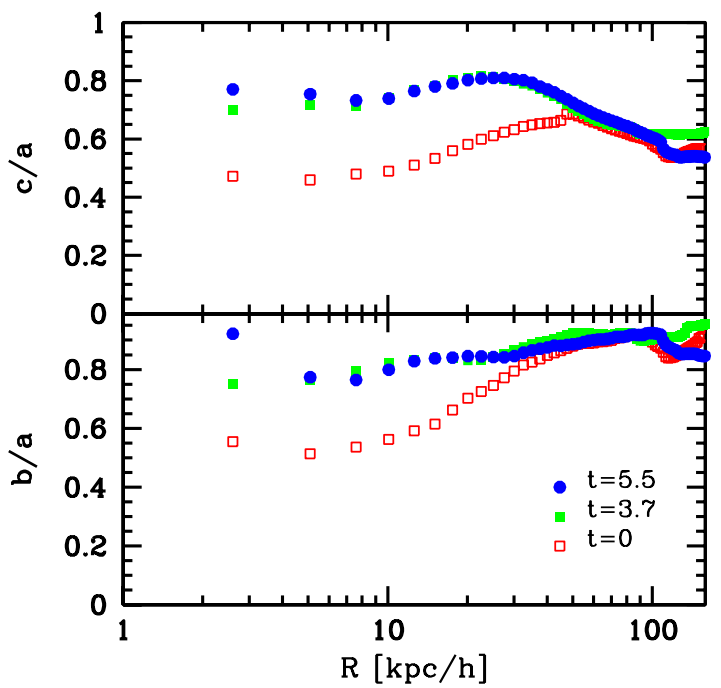

FIG. 6. - Radial dependence of the intermediate to major axis $(b / a)$ (bottom panel) and minor to major axis $(c / a)$ (top panel) ratios of ellipsoids fitted to the halo when a disk is included.

effect of the disk is to reduce the number of substructures and the masses of those that survive already after 3.7 Gyrs. Moreover, with time the disk also compresses the dark matter distribution in the center, further contributing to subhalo heating and accelerating the mass loss of these systems. A measurement of the halo radial dark matter density profile showed that the halo contracts and becomes denser by a factor of 2 within the inner regions owing to the gravitational potential of the disk. We also note that the presence of a disk makes the inner parts of the dark matter halo rounder, an effect that we further quantify below.

We can identify three physical mechanisms which cause substructures to lose mass and to eventually be disrupted. First, as these objects orbit within the halo-galaxy system, they will be tidally stripped. The contribution of the halo to this process has been included in previ- 
ous studies (Hayashi et al. 2003; Kazantzidis et al. 2004b; Read et al. 2006; Peñarrubia et al. 2008; Muñoz et al. 2008) but generally not that from the luminous galaxy (but see Taylor \& Babul 2001). Moreover, the adiabatic contraction of the halo owing to the disk enhances this process, an effect that is not accounted for in dark matter only simulations.

The impact of this effect can be estimated crudely by assuming that the orbiting substructure is truncated at some tidal radius $r_{t}$ where the differential tidal force of the halo-galaxy system is equal to the gravitational attraction of the satellite. For non-circular orbits the tidal radius is such that the average density of the satellite within it is proportional to the mean density of the Galaxy at $R_{p}$, the pericentric distance: $\rho_{\text {sub }}\left(r_{t}\right) \propto \rho_{\text {gal }}\left(R_{p}\right)$. N-body simulations confirm that subhalos are truncated at the radius given by $r_{t}$ (e.g. Springel et al. 2008a), but we expect that this process will be dominant only for satellites orbiting with large pericentric distances.

Second, the tidal field of the dark matter halo causes tidal heating of the particles in a satellite galaxy. This process is most efficient for satellites on eccentric orbits (Gnedin et al. 1999), which are the majority according to numerical simulations, whereas it vanishes for systems on circular orbits. Within $\sim 20 \mathrm{kpc}$ of the Milky Way center this process destroys substructures more rapidly than tidal stripping, owing to the strong tides that occur during close passages of the central dark matter cusp. We hence may also call this process halo shocking.

Third, when an object plunges through the dense baryonic disk, it will be subject to a process known as disk shocking (Ostriker et al. 1972; Binney \& Tremaine 1987) which can, in some circumstances, produce a much stronger response than tidal stripping (Taylor \& Babul 2001). This process is not accounted for in dark matter only simulations, and its relevance for the evolution of the substructure abundance has largely been ignored thus far. We will hence focus on this process in what follows.

Whereas a slowly varying tidal field can strip loosely bound material through tidal stripping, a rapidly varying gravitational field, such as that arising when a body passes through a galactic disk, will induce gravitational shocks that add energy to an object, changing its structure and accelerating mass loss. For an object orbiting in the inner regions of a galaxy on an orbit inclined with respect to the disk, disk shocking may indeed dominate the mass loss. This process is known to influence the structure of globular star clusters (Ostriker et al. 1972), and can be more significant for dark matter substructures. In the solar neighborhood, the galactic disk has a mass density $\rho \sim 0.2 \mathrm{M}_{\odot} \mathrm{pc}^{-3}$ and the vertical scale-height with which the density decreases with distance above or below the midplane is roughly $350 \mathrm{pc}$ (Binney \& Tremaine 1987). A clump of dark matter with mass of $\mathrm{M} \approx 10^{7} \mathrm{M}_{\odot}$ and halfmass radius $r_{1 / 2} \approx 1 \mathrm{kpc}$ has a mean density within $r_{1 / 2}$ of order $\rho \sim 0.002 \mathrm{M}_{\odot} \mathrm{pc}^{-3}, 1000$ times less than that of either the Milky Way disk locally or a globular cluster with $\mathrm{M} \approx 10^{6}$ $\mathrm{M}_{\odot}$ and $r_{1 / 2} \approx 10 \mathrm{pc}$. Therefore, disk shocking can be more important for the dynamics of dark matter substructures than for globular clusters.

Indeed, this suggestion is supported by an additional consideration. The characteristic internal velocities for a bound, virialized system are $\left\langle v^{2}\right\rangle \sim 0.4 G M / r_{1 / 2}$ (Spitzer 1969; Binney \& Tremaine 1987), typically $\sim 7.5 \mathrm{~km} \mathrm{~s}^{-1}$ for a globular cluster, and only $1.4 \mathrm{~km} \mathrm{~s}^{-1}$ for a clump of dark matter of this mass. When an object passes through a disk of vertical extent $2 z$, its constituents will move a distance $\Delta r \sim 2 z \sqrt{\left\langle v^{2}\right\rangle} / V_{\perp}$ along their orbits, where $V_{\perp} \sim 170 \mathrm{~km} \mathrm{~s}^{-1}$ is the mean vertical speed for typical inclined orbits through the disk of the Milky Way (Binney \& Tremaine 1987). Stars in a globular cluster will thus move a distance $\Delta r \sim 50 \mathrm{pc}$ during a disk crossing, implying that tightly bound stars will be protected by adiabatic invariance (Binney \& Tremaine 1987), and that only the outer, more loosely bound material will be affected. However, because the internal velocities characteristic of mass clumps of dark matter are lower, the particles within these objects will move smaller distances along their orbits, $\Delta r \sim 6 \mathrm{pc}$. This is much less than the half-mass radii of these substructures; therefore energy can be efficiently deposited into the bulk of the mass of such an object by disk shocking, in some cases leading to its complete disruption.

A substructure will acquire from a disk passage an energy per unit mass of order $\Delta E \sim 2\left\langle r^{2}\right\rangle g_{z}^{2} / 3 V_{\perp}^{2}$, where $g_{z}$ is the local gravitational acceleration above and below the disk. We note that the second moment $\left\langle r^{2}\right\rangle$ of the radial particle distribution of a subhalo is tightly correlated with its half-mass radius $r_{1 / 2}$; from the Aquarius $\mathrm{N}$-body simulations we find $\left\langle r^{2}\right\rangle \simeq\left(1.84 r_{1 / 2}\right)^{2}$. The heating energy $\Delta E$ is to be compared with the total gravitational binding energy of the object, which we can for example estimate according to $E_{b} \approx$ $-0.28 G M^{2} / r_{1 / 2}$, by fitting the density profile to a Hernquist (1990) model, although this choice makes little difference to our argument. An alternative is to assume virial equilibrium and estimate the binding energy from the total kinetic energy in random motions of the bound subhalo, i.e. $E_{b} \approx M\left\langle v^{2}\right\rangle / 2$.

For a substructure of mass of $10^{7} \mathrm{M}_{\odot}$, we find $\Delta E \sim E_{b}$ per orbit at $10 \mathrm{kpc}$ from the center. If the disk mass density declines exponentially with radius, then a corresponding estimate gives $\Delta E \approx 0.1 E_{b}$ per orbit at a distance of $20 \mathrm{kpc}$ from the center. Thus, complete disruption from disk shocking alone could occur at or within the solar radius during a single orbit, whereas $\approx 10$ orbits would be required to disrupt a substructure orbiting at a distance of $20 \mathrm{kpc}$. For objects passing through the disk in the solar vicinity on modestly inclined or eccentric orbits, the azimuthal period is $T_{\psi} \sim 2 \times 10^{8}$ years, implying a disruption timescale of order $\sim 2 \times 10^{9}$ years. This should be regarded as an upper limit because tidal stripping will accelerate mass loss when the substructure is above or below the disk, and because this estimate ignores the reduction in binding energy following each disk shock. These calculations may overestimate the rate of depletion for the subhalos with the half mass radius larger than the disk thickness. In this case the maximum amount of energy a substructure can receive per unit mass will be limited by the region of the substructure that is compressed/shocked at a given time which is of the order of disk thickness. Thus, the overall effect of disk shocking for subhalos larger than $10^{7}$ might be overestimated in this model.

A similar estimate applied to the more massive dark matter substructures in our simulations suggests that the disruption timescale for these objects is also short near the disk, explaining the rapid depletion of substructures seen in the inner regions of Figure 1. Indeed, in the simulation shown in Figure 1 with a disk, only one subhalo with maximum circular velocity larger than $10 \mathrm{~km} \mathrm{~s}^{-1}$ survives within $30 \mathrm{kpc}$ of the center of the galaxy. As it happens, this is in agreement with the one known Milky Way satellite on such a tightly bound orbit, Sagittarius at $24 \pm 2 \mathrm{kpc}$. Thus, it does not appear that there is an inner missing satellite problem in the Milky Way. 


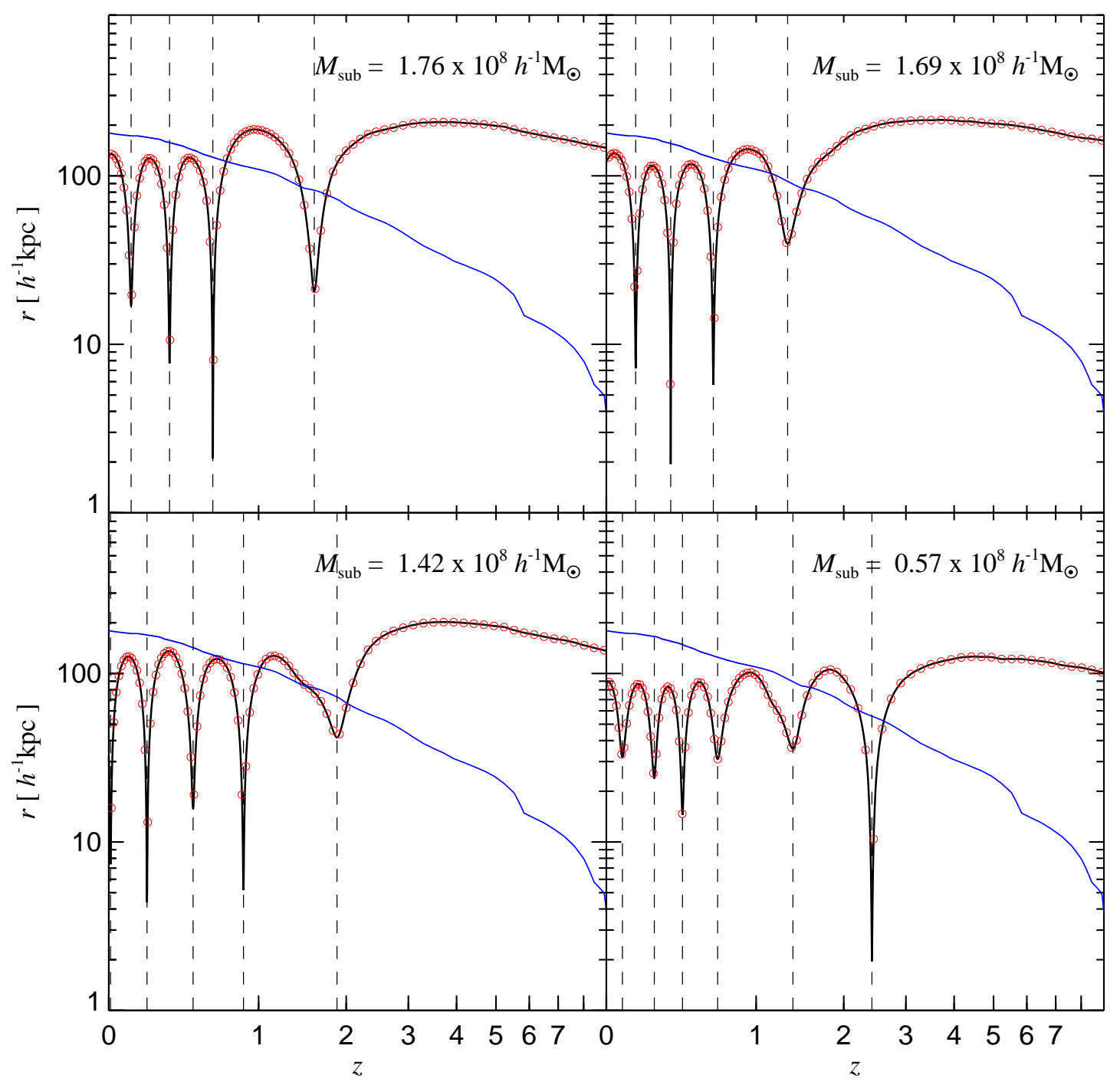

FIG. 7.- Examples of radial orbits for four surviving substructures in the Aq-A-2 simulation of Springel et al. (2008a). In each panel, the solid black line shows the physical distance of the substructure center to the center of the main progenitor halo of the forming Milky-Way sized halo. The virial radius of this growing main halo is shown as a blue line. The red circles mark the output times used in the analysis; the actual orbit is constructed with an interpolation technique based on these output times. The thin vertical lines mark the pericentric passages that are identified by our orbit tracking technique.

In Figure 5 we show the efficiency of the different processes causing mass loss and destruction of satellites as a function of the pericentric distances of their orbits. For a substructure orbiting within $20 \mathrm{kpc}$ of the galactic center the halo shocking process is stronger than tidal stripping. However, at slightly smaller radius, disk shocking becomes the dominant destruction process overall. Because of the presence of a disk in the inner regions, the Galactic halo contracts and becomes slightly denser in the inner regions, further enhancing the impact of tidal heating and stripping, although we note that our estimates in Figure 5 do not account for this.

\subsection{Halo shape}

Finally, we also note that the dark halo in Figure 1 appears to be more spherical when the disk is included (in particular, the middle and third columns of the bottom row) as compared to the simulation without a disk (top row), in qualitative agreement with previous studies which have tried to include baryonic material in live halos (Springel et al. 2004;
Kazantzidis et al.2004a; Maccio’ et al. 2007; Debattista et al. 2008; Abadi et al. 2009). Indeed, the halo responds to the presence of the disk by becoming significantly more spherical. This is quantified in Figure 6 where we measure the radial dependence of axial ratios of ellipsoidal surfaces of the halo as a function from the halo center when a disk is included. The panels in Figure 6 display the intermediate to major axis $(b / a)$ (bottom panel) and minor to major axis ratio $(c / a)$ (top panel) of the halo measured at different distances from the center. The presence of the disk turns the halo from a triaxial to more spherical object within $30-40 \mathrm{kpc}$ of the center, already after 3.7 Gyrs (the disk is fully in place after 2 Gyrs). The halo shape is not independent of radius. Indeed, this halo becomes more round with a baryonic component only within $30-40 \mathrm{kpc}$, roughly the size of the disk and it is not affected as far out the virial radius. Hence, our model suggests that halos around disk galaxies should be round only within the regions occupied by the disk and not necessarily far beyond that. 


\section{DISK PASSAGES IN COSMOLOGICAL SIMULATIONS}

In order to extend the dynamic range of the masses of substructures for which we can study the disk-shocking effect, we follow the orbits of subhalos in one of the high-resolution numerical realizations of Milky Way sized halos calculated in the 'Aquarius' project (Springel et al. 2008b; Navarro et al. 2008). In this suite of dark matter only simulations, extensive resolution studies for 6 different halos of mass $\sim 10^{12} \mathrm{M}_{\odot}$ were carried out, dark matter halos and substructures were found at a large number of output times, and detailed merger history trees for all these halos and subhalos were constructed. Using these trees allows us to accurately follow the orbits of individual subhalos and to determine how often they are expected to cross a fiducial disk assumed to be present in the galaxy-sized halos.

We here focus most of our analysis on the 'Aq-A-2' simulation, which has a mass resolution of $10^{4} h^{-1} \mathrm{M}_{\odot}$, and a virial mass of $M_{200}=1.34 \times 10^{12} h^{-1} \mathrm{M}_{\odot}$ within $R_{200}=180 h^{-1} \mathrm{kpc}$ (the radius enclosing a mean overdensity of 200 relative to the critical density) for its final Milky Way-sized halo. Based on the substructure catalogs and merger trees, we construct continuous orbits for all substructures by interpolating the subhalo trajectories with the discrete set of coordinates and velocities available for the output times. We use reconstructed orbits based on 128 outputs between redshifts $z \sim 20$ and $z=0$ for the analysis shown here, but we have checked that using up to 1024 outputs instead does not change the estimated disk encounter parameters in any significant way; i.e. the time resolution of 128 outputs is sufficient to obtain sufficiently accurate orbital tracks for the purposes of this analysis.

In Figure 7] we show examples of radial orbits for four typical substructures with mass of around $10^{8} h^{-1} \mathrm{M}_{\odot}$ that end up within the virial radius of the final halo. We clearly see that the subhalos make several orbits, sometimes reaching quite small pericentric distances to the halo center. Note that even substructures that presently have a comparatively large distance from the center may have had a close encounter with the halo center at some earlier time. For example, the subhalo shown in the bottom right panel has now a distance of $r \sim 90 h^{-1} \mathrm{kpc}$, but it passed by the center at a distance of less than $2 h^{-1} \mathrm{kpc}$ as early as $z \simeq 2.5$. Such close passages at small distances to the center may then lead not only to significant halo shocking (which the substructures shown in this plot in fact did survive) but also to disk shocking.

In order to obtain an estimate for the cumulative effect of the latter, we examine the orbits of all substructures that end up inside the virial radius at $z=0$ and which have a mass above $M_{\text {sub }}=10^{6} h^{-1} \mathrm{M}_{\odot}$ at the final time. For each passage through a fiducial plane containing the disk of the main halo (say the $x y$-plane, but choosing a different orientation gives consistent results), we register the distance $R$ to the center, the velocity $V_{\perp}$ perpendicular to the disk, the subhalo's current half-mass radius $r_{1 / 2}$ and its squared 3D velocity dispersion $\left\langle v^{2}\right\rangle$. This allows us to characterize the strength of the diskshocking as

$$
\frac{\Delta E}{E}=\frac{\left(1.84 r_{1 / 2}\right)^{2}[4 \pi G \Sigma(R)]^{2}}{3\left\langle v^{2}\right\rangle V_{\perp}^{2}},
$$

where we assumed the infinite sheet approximation to relate the disk's surface mass density $\Sigma(R)$ to the vertical gravitational field above and below the disk. For systems that experience multiple disk transitions, we simply add up the different $\Delta E / E$ values. A significant disk shocking effect can be expected when $\Delta E / E$ reaches values of order unity. We shall assume that $\Delta E / E \geq 1$ implies certain disruption, but we note that also smaller values, $\Delta E / E \gtrsim 0.1$, should lead to significantly accelerated mass loss and earlier destruction.

The distribution of $\Delta E / E$ values obtained in this way is broad, with many subhalos having very small values, suggesting that for them disk shocking is unimportant. However, we find that about $14.1 \%$ of the subhalos with mass above $M_{\text {sub }}=10^{6} h^{-1} \mathrm{M}_{\odot}$ have $\Delta E / E$ larger than 1.0 , and $24.2 \%$ have a value larger than 0.1 .

In Figure 8, we show the estimated impact of disk-shocking in terms of the cumulative radial count of subhalos, comparing the counts of all subhalos to those where systems with $\Delta E / E \geq 1.0$ or $\Delta E / E \geq 0.1$ are omitted. Interestingly, the cumulative effect is clearly stronger in the inner than in the outer parts. In fact, for the small mass subhalos that dominate the $M_{\text {sub }} \geq h^{-1} 10^{6} \mathrm{M}_{\odot}$ sample, the reduction at radii of $\sim 30 \mathrm{kpc}$ reaches a factor of $\sim 3-4$, whereas it is only about $25 \%$ at the virial radius. We also include results restricted to the more massive subhalos $M_{\text {sub }}=10^{8} h^{-1} \mathrm{M}_{\odot}$ in Figure 8 . This shows a qualitatively similar trend, but with poorer statistics. We find that the more massive subhalos tend to be less affected by disk shocking, which can be understood based on their more recent infall time and hence smaller number of passages through the disk.

We note that the precise strength of the disk shocking effect depends, of course, on the adopted disk model. In the results above, we have assumed a fixed exponential disk with scale radius $5 h^{-1} \mathrm{kpc}$ and with mass equal to $10 \%$ of the virial mass of the final halo. Alternatively, we have explored models where we assumed that the disk mass grows in proportion to the virial mass of the halo, and the disk size scales with the halo's virial radius. This moderately reduces the strength of the effect; we then find that $17.1 \%$ instead of $24.2 \%$ of the subhalos reach a value $\Delta E / E \geq 0.1$.

Another very interesting question is whether disk shocking in progenitor systems other than those of the main halo may be important for the final substructure population. Note that the results above have only estimated disk shocking from the central disk, but perhaps many substructures fall in as part of groups, in which case they could already have experienced severe tidal shocks owing to the baryonic structures in these groups.

To investigate this idea, we first ask the question of what is the fraction of substructures that have fallen in as a subhalo within a larger system, as opposed to being accreted as a main halo on their own. This can be readily addressed with the merger trees of the Aquarius halos. In Figure 9, we show the fraction of subhalos that were accreted as a substructure of an infalling group, as a function of their present day mass for all subhalos in the virial radius. We compare results for simulations of the same halo at different numerical resolutions, finding good convergence. We see that the fraction of substructures that come in already as a subhalo in a larger group is actually quite small, only about $10-20 \%$ over the mass range considered, with a slight trend to increase towards smaller masses (see also Ludlow et al. 2009). In hindsight this is perhaps not too surprising, because for any given mass, there are always more main halos as genuine subhalos, and the merger hierarchy in cold dark matter models is actually surprisingly 'shallow' (Angulo \& White 2009).

However, this also means that disk shocking in progenitor systems different from the progenitors of the main halo 
(which form the 'main stem' of the merger tree) is unlikely to be very important.

We explicitly confirm this by extending our estimates of disk shocking to include all progenitor groups and not only the main halo. To this end, we extract disk passage parameters also when the reconstructed orbit of a subhalo passes by the center of a main halo different from the main progenitor of the primary galaxy. For simplicity, we assume that there are also disks present in these secondary progenitor systems, and that they always have a disk mass equal to $10 \%$ of their current virial mass, with a size scaled in proportion to their virial radius. We then find that only a relatively small number of additional subhalos are predicted to be strongly affected by disk shocking. In particular, when using the $\Delta E / E \geq 0.1$ criterion, the number of affected subhalos goes up from $24.2 \%$ to $24.8 \%$ when the disk in the main halo is kept fixed, while it goes up from $17.1 \%$ to $17.8 \%$ when the disk is assumed to always scale with halo size.

Comparing the results from this analysis of substructure orbits in the Aquarius halos to our direct disk-growth simulations, we find in general reasonable agreement. In particular, the effect estimated from the Aquarius simulations for the inner regions of the halo is of comparable magnitude to that found in our disk-growth experiments, even though it appears a bit weaker overall. But this is not unexpected. For one, the disk-growth simulations experience a central compression of the dark matter halo which causes an additional increase in the efficiency of halo shocking, an effect that we have not taken into account in the analysis in the present section. In addition, disk shocking will accelerate the mass loss of surviving subhalos, which is also an effect that we have not included here. We emphasize that the calculations in this section are based on analytic estimates and involve several approximations. A self-consistent analysis of the orbital properties of subhalos and their mass loss using numerical simulations when the disk is included in a Milky Way halo is required to study these issues in more detail.

\section{DISCUSSION AND CONCLUSION}

Tidal halo shocking and disk shocking are dominant effects compared to tidal stripping for fairly eccentric orbits and have not been included in all previous simulations of dark satellites orbiting luminous galaxies like the Milky Way. Our work shows that these processes can be efficient at depleting the satellite population within $\sim 30 \mathrm{kpc}$ of the Milky Way center.

While the effects we model in our simulations can extend further than the radial scale-lengths of disks, because many substructures are on moderately radial orbits, our calculations do not resolve the "missing satellite problem" on larger spatial scales. In principle, the processes we have investigated here could propagate from smaller to larger mass scales if one accounted for visible galaxies in the substructures that merged to form the Mily Way halo, driving pre-destruction of substructures within subhalos and leading to a stronger depletion of satellites in the outer regions of large halos. However, our estimates indicate that most substructures are accreted as main halos and not as subhalos, limiting the overall importance of this effect. We do note, however, that if luminous satellite galaxies in the Milky Way formed more efficiently in subhaloes accreted as part of a Magellanic Group, as proposed by D'Onghia \& Lake (2008), then it would not be surprising that most substructures would remain dark, even if they are not destroyed.

Our findings have interesting implications for understand-

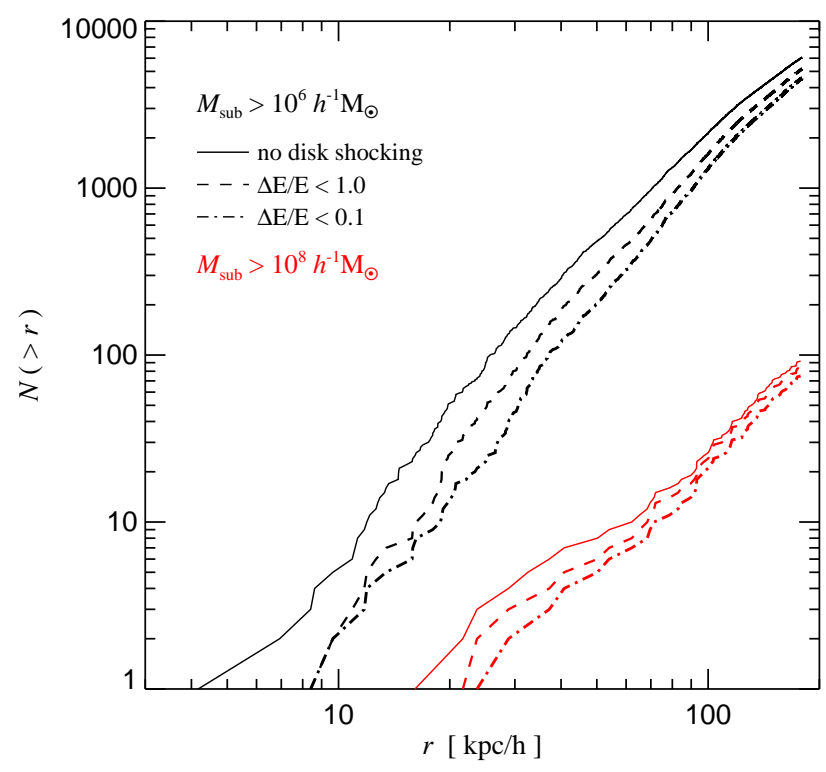

FIG. 8.- Cumulative radial distribution of satellites with mass larger than $10^{6} h^{-1} \mathrm{M}_{\odot}$ (black lines) and larger than $10^{8} h^{-1} \mathrm{M}_{\odot}$ (red lines) in the Aq-A-2 high-resolution simulation of the Aquarius project. The cumulative count is shown for the original subhalo population (solid lines), and when subhalos are excluded for which the estimated disk shocking on their orbits exceeds $\Delta E / E=1.0$ (dashed) and $\Delta E / E=0.1$ (dot-dashed), respectively.

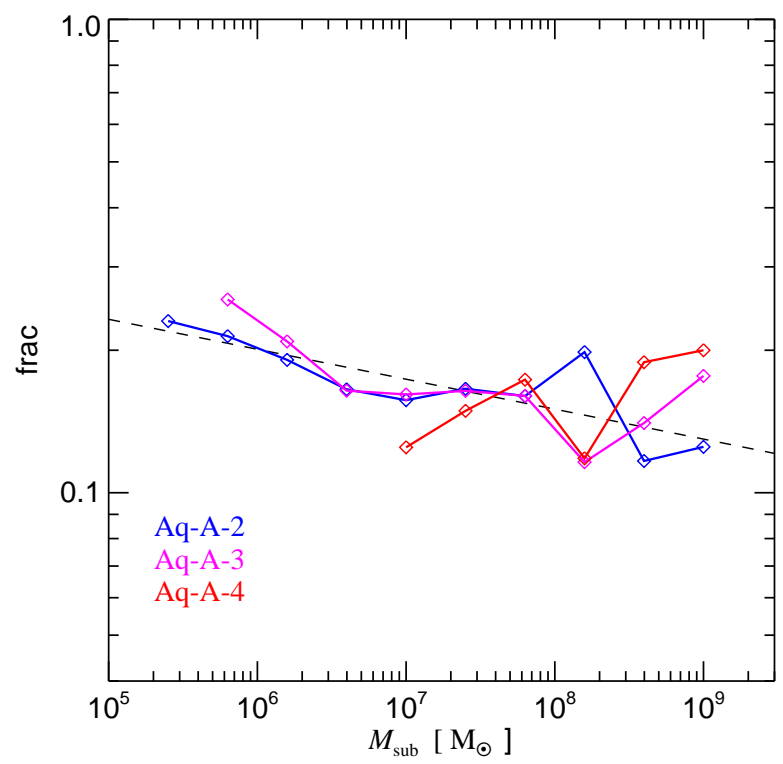

FIG. 9.- Fraction of substructures present in the main halo at $z=0$ that were accreted as subhalos of another infalling structure, as opposed to being accreted as a main halo. We compare results at different numerical resolutions. For runs Aq-A-3 and Aq-A-4, the mass resolutions are factors of 3.6 and 30 times worse than in our default simulation Aq-A-2, respectively. The dashed-line marks a power-law fit to the results for Aq-A-2.

ing the nature of spiral structure in disk galaxies. Recent simulations have shown that dark satellites impacting a disk could play an important role in generating the multi-armed, global spirals seen in disk galaxies. Our work demonstrates that the majority of the satellites are depleted in a short time when they pass through the disk. This raises the question of whether an early impact of satellites inside, or later impacts outside, the disk could still provide a source of perturbations 
capable of maintaining global spiral patterns in disk galaxies, or whether a different source is required. Interestingly, an alternative source might be molecular gas clumps with masses of $10^{6}-10^{7} M_{\odot}$ which are preferentially found close to the spiral arms in the Milky Way or infalling halo clouds which have similar masses (Kereš \& Hernquist 2009).

Although we do not account for the presence of live disks we do not expect the passages of the tightly bound substructures through the disk before these objects are destroyed to have a significant effect on disk kinematics. Hopkins et al. (2008) showed that the heating done to disks by smaller perturbers is second order in the mass ratio, so heating of the disk by substructures in the mass range we have explored is likely to be unimportant, as also shown by Velazquez \& White (1999) using N-body simulations.

Another implication of our work is that the depletion of clumps within a few dozen of kiloparsecs from the Milky Way center might lead to a revision of the mass fraction embedded in substructures as implied by gravitational lensing. These observations have been done for early-type galaxies where there is no disk and might lead to different values when applied to late-type/disk galaxies. Furthermore, recent work estimating the flux ratio anomalies in gravitational lensing owing to the presence of substructure points out that the inner subhalo abundance predicted by CDM-only simulations appears actually slightly too low to account for these anomalies (Xu et al. 2009). As we have shown here, because baryonic processes can reduce the inner substructure abundance significantly, it might turn out that this problem is even more severe. On the other hand, there are cases where these anomalous flux ratios are likely the result of visible substructures. In particular, in CLASS B2045+265, one of the most extreme anomalous flux ratio systems, McKean et al. (2007) find a small dwarf galaxy companion which is sufficient to fully explain the observed flux ratios without the need for any additional dark substructures.

Diemand et al. (2008) and Springel et al. (2008b) have computed the dark matter annihilation signal from the galactic substructures and from the smooth dark matter halo of the Milky Way using very high resolution cosmological Via Lactea II and Aquarius simulations, respectively. It was pointed out (Springel et al. 2008b) that the signal-to-noise for detection of dark matter annihilation from the galactic center is in general much larger than for any of the substructures, but in case substructures are detectable, their typical distance from the Solar circle is predicted to be around $20 \mathrm{kpc}$. Hence this detectable substructure population would be strongly affected by the disc shocking effect. Other models of gammaray emission built on the Via Lactea II simulation predict that the number of dark clumps detectable from the Fermi-LAT is between 1 and 10 in five years of operation, for an optimistic dark matter scenario (Pieri et al. 2009b), but see also Kuhlen et al. (2009) for an even larger estimate assuming an additional strong boost of the annihilation rate due to unresolved dark clumps. Our results reduce these values by a factor of 3 for subhalos of mass $10^{7} \mathrm{M}_{\odot}$, and by a factor of 2 for larger masses. This strengtens the expectation that detection of the dark matter annihilation signal from the galactic center should be easier than from nearby substructures (Springel et al. 2008b; Pieri et al. 2009a).

Ultimately disk shocking predicts that luminous satellite galaxies orbiting through galactic disks should leave long tails of stars and can be disrupted in a few Gyrs, a timescale much shorter than the tails live. This possibility is supported by recent observations of loops of tidal tails around NGC 5907, which may be the remains of a ghost dwarf galaxy that has not been found (Martínez-Delgado et al.|2008).

This research was partly supported by the EU Marie Curie Intra-European Fellowship under contract MEIF-041569. D.K. acknowledges supports by ITC fellowship. Numerical simulations were performed on the Odyssey supercomputer at Harvard University. The simulations of the Aquarius project were carried out at the Leibniz Computing Center and the Computing Center of the Max-Planck-Society in Garching, Germany.

\section{REFERENCES}

Abadi, M. G., Navarro, J. F., Fardal, M., Babul, A., \& Steinmetz, M. 2009, ArXiv:0902.2477

Angulo, R. E. \& White, S. D. M. 2009, ArXiv:0906.1730

Bett, P., Eke, V., Frenk, C. S., Jenkins, A., Helly, J., \& Navarro, J. 2007, MNRAS, 376, 215

Binney, J. \& Tremaine, S. 1987, Galactic dynamics, ed. J. Binney \& S. Tremaine

Bouchet, F. R. \& Hernquist, L. 1988, ApJS, 68, 521

Bryan, G. L. \& Norman, M. L. 1998, ApJ, 495, 80

Debattista, V. P., Moore, B., Quinn, T., Kazantzidis, S., Maas, R., Mayer, L., Read, J., \& Stadel, J. 2008, ApJ, 681, 1076

Diemand, J., Kuhlen, M., Madau, P., Zemp, M., Moore, B., Potter, D., \& Stadel, J. 2008, Nature, 454, 735

Diemand, J., Moore, B., \& Stadel, J. 2005, Nature, 433, 389

Dolag, K., Borgani, S., Murante, G., \& Springel, V. 2008, ArXiv:0808.3401

D’Onghia, E. \& Lake, G. 2008, ApJ, 686, L61

D’Onghia, E. \& Navarro, J. F. 2007, MNRAS, 380, L58

Dubinski, J., Gauthier, J.-R., Widrow, L., \& Nickerson, S. 2008, in Astronomical Society of the Pacific Conference Series, Vol. 396, Astronomical Society of the Pacific Conference Series, ed. J. G. Funes \& E. M. Corsini, 321

Ghigna, S., Moore, B., Governato, F., Lake, G., Quinn, T., \& Stadel, J. 2000, ApJ, 544, 616

Gnedin, O. Y., Hernquist, L., \& Ostriker, J. P. 1999, ApJ, 514, 109

Hayashi, E., Navarro, J. F., Taylor, J. E., Stadel, J., \& Quinn, T. 2003, ApJ, 584,541

Hernquist, L. 1990, ApJ, 356, 359

Hernquist, L. \& Katz, N. 1989, ApJS, 70, 419
Hopkins, P. F., Hernquist, L., Cox, T. J., Younger, J. D., \& Besla, G. 2008, ApJ, 688, 757

Johnston, K. V., Spergel, D. N., \& Hernquist, L. 1995, ApJ, 451, 598

Julian, W. H. \& Toomre, A. 1966, ApJ, 146, 810

Kazantzidis, S., Kravtsov, A. V., Zentner, A. R., Allgood, B., Nagai, D., \& Moore, B. 2004a, ApJ, 611, L73

Kazantzidis, S., Mayer, L., Mastropietro, C., Diemand, J., Stadel, J., \& Moore, B. 2004b, ApJ, 608, 663

Kazantzidis, S., Zentner, A. R., Kravtsov, A. V., Bullock, J. S., \& Debattista, V. P. 2009, ArXiv:0902.1983

Kereš, D. \& Hernquist, L. 2009, ApJ, 700, L1

Klypin, A., Kravtsov, A. V., Valenzuela, O., \& Prada, F. 1999, ApJ, 522, 82

Komatsu, E., Dunkley, J., Nolta, M. R., Bennett, C. L., Gold, B., Hinshaw, G., Jarosik, N., Larson, D., Limon, M., Page, L., Spergel, D. N., Halpern, M., Hill, R. S., Kogut, A., Meyer, S. S., Tucker, G. S., Weiland, J. L., Wollack, E., \& Wright, E. L. 2009, ApJS, 180, 330

Kravtsov, A. V. 2009, ArXiv:0906.3295

Kuhlen, M., Madau, P., \& Silk, J. 2009, Science, 325, 970

Kuzmin, G. G. 1956, Astron. Zh., 33, 27

Ludlow, A. D., Navarro, J. F., Springel, V., Jenkins, A., Frenk, C. S., \& Helmi, A. 2009, ApJ, 692, 931

Maccio', A. V., Sideris, I., Miranda, M., Moore, B., \& Jesseit, R. 2007, ArXiv e-prints

Martínez-Delgado, D., Peñarrubia, J., Gabany, R. J., Trujillo, I., Majewski, S. R., \& Pohlen, M. 2008, ApJ, 689, 184

McKean, J. P., Koopmans, L. V. E., Flack, C. E., Fassnacht, C. D., Thompson, D., Matthews, K., Blandford, R. D., Readhead, A. C. S., \& Soifer, B. T. 2007, MNRAS, 378, 109 
Moore, B., Ghigna, S., Governato, F., Lake, G., Quinn, T., Stadel, J., \& Tozzi, P. 1999, ApJ, 524, L19

Muñoz, R. R., Majewski, S. R., \& Johnston, K. V. 2008, ApJ, 679, 346

Navarro, J. F., Ludlow, A., Springel, V., Wang, J., Vogelsberger, M., White, S. D. M., Jenkins, A., Frenk, C. S., \& Helmi, A. 2008, ArXiv:0810.1522

Ostriker, J. P., Spitzer, L. J., \& Chevalier, R. A. 1972, ApJ, 176, L51

Peñarrubia, J., Navarro, J. F., \& McConnachie, A. W. 2008, ApJ, 673, 226

Pieri, L., Lavalle, J., Bertone, G., \& Branchini, E. 2009a, ArXiv e-prints

Pieri, L., Pizzella, A., Corsini, E. M., Dalla Bontà, E., \& Bertola, F. 2009b, A\&A, 496, 351

Read, J. I., Wilkinson, M. I., Evans, N. W., Gilmore, G., \& Kleyna, J. T. 2006 , MNRAS, 367,387

Spitzer, L. J. 1969, ApJ, 158, L139

Springel, V. 2005, MNRAS, 364, 1105

Springel, V., Wang, J., Vogelsberger, M., Ludlow, A., Jenkins, A., Helmi, A., Navarro, J. F., Frenk, C. S., \& White, S. D. M. 2008a, MNRAS, 391, 1685

Springel, V., White, S. D. M., Frenk, C. S., Navarro, J. F., Jenkins, A., Vogelsberger, M., Wang, J., Ludlow, A., \& Helmi, A. 2008b, Nature, 456, 73
Springel, V., White, S. D. M., \& Hernquist, L. 2004, in IAU Symposium, Vol. 220, Dark Matter in Galaxies, ed. S. Ryder, D. Pisano, M. Walker, \& K. Freeman, 421

Springel, V., White, S. D. M., Jenkins, A., Frenk, C. S., Yoshida, N., Gao, L., Navarro, J., Thacker, R., Croton, D., Helly, J., Peacock, J. A., Cole, S., Thomas, P., Couchman, H., Evrard, A., Colberg, J., \& Pearce, F. 2005, Nature, 435, 629

Springel, V., White, S. D. M., Tormen, G., \& Kauffmann, G. 2001, MNRAS, 328,726

Taylor, J. E. \& Babul, A. 2001, ApJ, 559, 716

Toomre, A. 1981, in Structure and Evolution of Normal Galaxies, ed. S. M. Fall \& D. Lynden-Bell, 111-136

Velazquez, H. \& White, S. D. M. 1999, MNRAS, 304, 254

White, S. D. M. \& Rees, M. J. 1978, MNRAS, 183, 341

Xu, D. D., Mao, S., Wang, J., Springel, V., Gao, L., White, S. D. M., Frenk, C. S., Jenkins, A., Li, G., \& Navarro, J. F. 2009, ArXiv:0903.4559 